\title{
THE FORMATION OF DISCONTINUITIES IN GAS FLOWS IN THE ISM AND ITS RELATION TO THE GALACTIC SYNCHROTRON RADIO EMISSION
}

\author{
V.G. BERMAN ${ }^{1}$, L.S. MAROCHNIK ${ }^{2}$, YU.N. MISHUROV ${ }^{1}$, \\ A.A. SUCHKOV ${ }^{1}$ \\ ${ }^{1}$ Rostov State University, Rostov-on-Don, USSR \\ ${ }^{2}$ Space Research Institute, Academy of Sciences, Moscow, USSR
}

\begin{abstract}
We show that large-scale motions of the interstellar gas, such as those associated with galactic density waves, easily develop, over a wide range of scales, shocks and discontinuities which are expected to generate turbulence. The latter is supposed to evoke diffusion of magnetic fields and cosmic rays on scales down to a few parsecs. We suggest that these processes may be of major importance in discussions of interconnections between the observed radio emission of the disks of spiral galaxies and the gas density distribution within them. In particular, we predict that the density of cosmic rays and magnetic field energy must be much less contrasted (on scales of $\sim 1 \mathrm{pc}$ and up to the scales of galactic shocks) than the gas density, hence the synchrotron radio emission is not as contrasted as is predicted under the hypothesis of a fully frozen-in magnetic field.
\end{abstract}

The interrelation between gas dynamics and magnetic fields in galactic disks is twofold. On the one hand, magnetic fields and cosmic rays, being frozen into the gas, tend to suppress the development of gas flows characterized by large contrasts of gas density, velocity, etc. On the other hand, the development of gas turbulence strongly enhances the diffusion of magnetic field, thus reducing its influence on gas motion. The gas and field become significantly decoupled, so the gas can develop large contrasts, with the magnetic field (and cosmic rays) only weakly reacting (and acting) on them.

One of the main problems here is the origin of the turbulence itself. A widely accepted view is that it is generated by shocks produced by supernovae explosions. We suggest another alternative, according to which the turbulence can be excited in a wide range of scales in gas flows connected with density waves. Here we show that such flows can easily develop shocks and discontinuities which may be regarded as onset of turbulence; on the other hand, these shocks and continuities are expected to represent the source of turbulence under the actual conditions in galactic disks.

We have considered an initially circular motion of gas in a disk where a gravitational field of a stellar density wave gradually arises; the 
gas is supposed to be cooled radiatively and to be heated by cosmic rays, which in thermal equilibrium produces the pressure-density relation of the van der Waals type (cf. Marochnik et al., 1983).

Figure 1 shows the distribution of gas density at three moments of time. It can be seen that the density grows in the potential well of the growing density wave, and there forms a galactic shock (upper panel). When the density on the shock front reaches the critical value $n_{c}^{4} f, a$ certain part of the gaseous mass here rapidly cools and contracts, with two contact discontinuities being formed at its boundaries (middle panel). The density and temperature suffer at the boundaries a jump of about two orders of magnitude, the pressure varies much less, the velocity remains almost continuous. The cooled mass, displayed in the middle panel as a density peak, is carried as a whole out of the shock front and moves downstream with the main flow. It is very tempting to interprete this process as a formation of a cool, dense cloud (of course, due to one-dimensional geometry, we cannot get a three-dimensional view of such a 'cloud' which may turn out, for example, to be in fact an

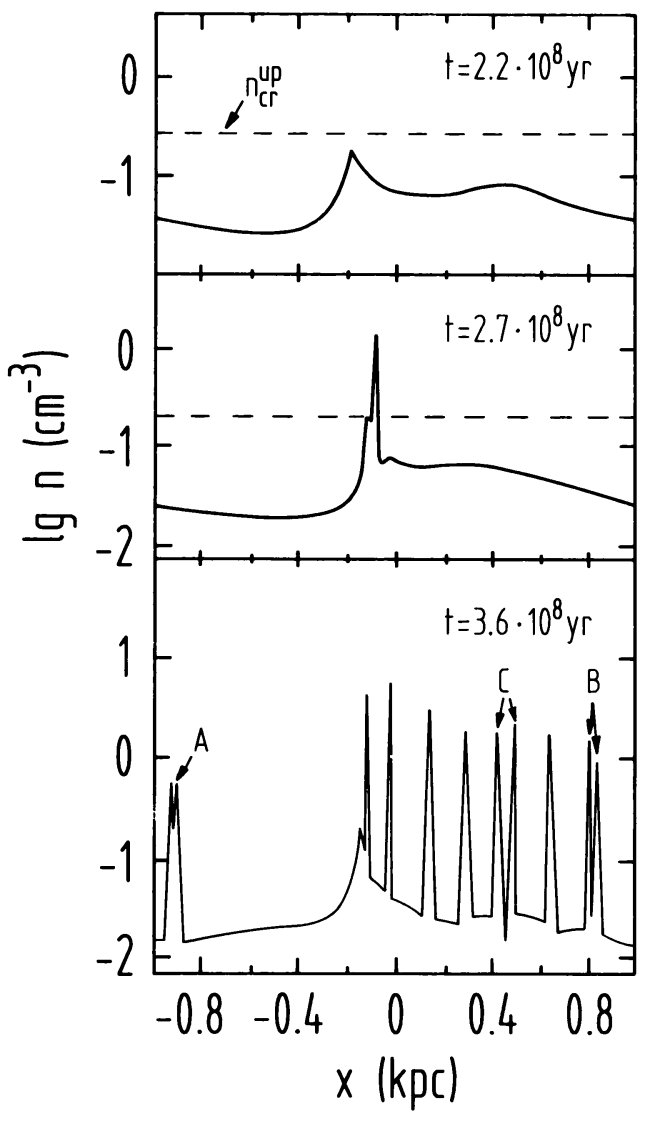

Figure 1. The profile of gas density at three moments of time, the $x$ coordinate is directed across the spiral arm, with $x=0$ corresponding to the minimum of the potential well of the twoarmed density wave. The profile is periodical in $x$, the period being $\sim 2 \mathrm{kpc}$. The gas moves through the potential well from left to right. The letters A, B, C indicate the inflating 'clouds' (see Figure 2). 
extended layer instead of a sphere).

Formation of the cloud reduces the pressure and density at the shock front, and some time is required for these parameters to reach the upper critical values again. After that the process is repeated and the second cloud is formed, then the third one and so on (lower panel). So the solution obtained visualizes that a galactic shock can really be that 'machine' which 'manufactures' clouds in galaxies (cf. Marochnik et al., 1983).

The density peaks seen in Figure 1 are in fact unresolved with respect to their true spatial structure. Their true size can be seen in Figure 2 where we show on a much smaller scale the evolution of a 'cloud' corresponding to one of the peaks from Figure 1. In case of the cloud shown in Figure 2 the density in the cloud centre at some moment has dropped below the value corresponding to the lower critical point of the equilibrium curve. As a result the temperature has rapidly jumped up here which has caused a rapid increase in pressure. This has led to a shock propagating outwards. The cloud inflates, forming a bubble of hot gas inside a dense, cold shell.



Figure 2. The smallscale structure of the density peak designated as ' $A$ ' in Figure 1 at $\mathrm{t}=3.644 \cdot 10^{8} \mathrm{yr}$.

Summarizing, we see that an initially uniform gas flow in the presence of a stellar density wave develops a large-scale galactic shock as well as small-scale contact discontinuities and shocks.

It is to be expected that the pressure of magnetic fields would soften these processes. At the same time, non-stationary shocks and contact discontinuities would undoubtedly generate miscellaneous waves, thus generating turbulence, and the turbulent diffusion of magnetic fields would reduce the effect of magnetic pressure on the development of density contrasts in the gas. Therefore it may be suggested that magnetic fields (and cosmic rays) do not influence as strong as they could (if fully frozen into the gas) the formation of shocks and clouds, and that the variation of magnetic field energy (and cosmic ray density) is significantly smaller than that of the gas density.

\section{Reference}

Marochnik, L.S., Berman, V.G., Mishurov, Yu.N. and Suchkov, A.A. (1983) Astrophys. Space Sci. 89, 177. 
MOUSCHOVIAS: In 1974 (Astron. Astrophys. 33, 73) Mouschovias, Shu and Woodward proposed that galactic shocks trigger the Parker instability and lead to the formation of cloud complexes, OB associations, and giant HII regions separated by regular intervals $\sim 1 \mathrm{kpc}$ along spiral arms. The thermal instability, which you have used to form clouds behind galactic shocks, is suppressed by a magnetic field as small as $1 \mu \mathrm{G}$ in all directions except parallel to $\vec{B}$ (see Field 1965, Ap.J. 142, 531). Moreover, the unstable wavelengths are limited by the product of the sound speed (usually $<10 \mathrm{~km} / \mathrm{sec}$ ) and the cooling time of the gas $\left(<10^{6} \mathrm{yr}\right.$ ); that is, they are less than $10 \mathrm{pc}$. Therefore, the amount of mass involved is less or much much less than $1 \mathrm{M}_{\odot}$. These are tiny cloudlets, not anything like the observed interstellar clouds (see review by Mouschovias 1981, in Fundamental Problems in the Theory of Stellar Evolution, eds. D. Sugimoto, D.Q. Lamb and D.N. Schramm, Reidel, Dordrecht, p. 27).

MAROCHNIK: Our numerical simulations show that the sizes of the clouds obtained are just of the order of $\ell \leqslant 10 \mathrm{pc}$. However, it turns out that the density of peaks which correspond to "clouds" obtained is of the order of $n \geqslant 10^{2} n_{0}$ (where $n_{0}$ is an initial value of the density). Hence the masses of the clouds (which we can estimate as $4 / 3 \pi(\ell / 2)^{3} m_{H} n$ ) are of the order of 50-100 M० under the condition $n_{0} \simeq 0.05-0.1 \mathrm{~cm}^{-3}$. The discrepancy between our numerical simulation and the estimates noted by you is not too clear. I believe that it is necessary to find an answer to this question.

KAHN: Most interstellar physicists no longer believe that soft cosmic rays have much to do with heating the interstellar gas. Also galactic dynamicists have severe doubts nowadays about shocks associated with spiral density waves, à la Lin and Roberts.

MAROCHNIK: Physical phenomena obtained in our numerical simulations are connected, generally speaking, almost only with the presence of the pressure-density relation of the van der Waals type. However, this relation is only an initial condition. The specific nature of the heating process is not essential for this numerical model as long as the van der Waals type pressure-density relation holds.

HEILES: There is not universal agreement that the filling factor of the hot gas is so large as to prevent ordinary shocks being generated by the spiral density wave. Even if it is so large, the density wave pushes individual clouds closer together, increasing their collision rate, which is in a sense equivalent to a shock.

MAROCHNIK: Of course, we have computed idealized versions of the interstellar gas flows across the density waves. We have suggested that these flows are homogeneous (a possible presence of the clouds at the onset is not taken into account). However, we show that under acceptable initial parameters, both global (galactic) and local (clouds), shocks can be formed. 